\title{
Prokaryotic expression of a novel mouse pro-apoptosis protein PNAS-4 and application of its polyclonal antibodies
}

\author{
P. Zhang ${ }^{1 *}$, C.T. Wang ${ }^{1 *}$, F. Yan ${ }^{1}$, L. Gou ${ }^{1}$, A.P. Tong ${ }^{2}$, F. Cai ${ }^{2}$, Q. Liं ${ }^{2}$ H.X. Deng ${ }^{1}$ \\ and Y.Q. Wei ${ }^{1}$
}

${ }^{1}$ State Key Laboratory of Biotherapy, West China Hospital, West China Medical School, ${ }^{2}$ School of Life Sciences, Sichuan University, Chengdu, Sichuan, China

Correspondence to: H.X. Deng, State Key Laboratory of Biotherapy, West China Hospital, West China Medical School, Sichuan University, Keyuan Road 4, Chengdu, Sichuan, 610041, China

Fax: +86-28-8516-4060. E-mail: evenforeven@yahoo.com.cn

\begin{abstract}
Mouse PNAS-4 (mPNAS-4) has 96\% identity with human PNAS-4 (hPNAS-4) in primary sequence and has been reported to be involved in the apoptotic response to DNA damage. However, there have been no studies reported of the biological functions of mPNAS-4. In studies conducted by our group (unpublished data), it was interesting to note that overexpression of mPNAS-4 promoted apoptotic death in Lewis lung carcinoma cells (LL2) and colon carcinoma cells (CT26) of mice both in vitro and in vivo. In our studies, mPNAS-4 was cloned into the pGEX-6P-1 vector with GST tag at N-terminal in Escherichia coli strain BL21(DE3). The soluble and insoluble expression of recombinant protein mPNAS-4 (rmPNAS-4) was temperature-dependent. The majority of rmPNAS-4 was insoluble at $37^{\circ} \mathrm{C}$, while it was almost exclusively expressed in soluble form at $20^{\circ} \mathrm{C}$. The soluble rmPNAS4 was purified by one-step affinity purification, using a glutathione Sepharose 4B column. The rmPNAS-4 protein was further identified by electrospray ionization-mass spectrometry analysis. The search parameters of the parent and fragment mass error tolerance were set at 0.1 and $0.05 \mathrm{kDa}$, respectively, and the sequence coverage of search result was $28 \%$. The purified rmPNAS-4 was further used as immunogen to raise polyclonal antibodies in New Zealand white rabbit, which were suitable to detect both the recombinant and the endogenous mPNAS-4 in mouse brain tissue and LL2 cells after immunoblotting and/or immunostaining. The purified rmPNAS-4 and our prepared anti-mPNAS-4 polyclonal antibodies may provide useful tools for future biological function studies for mPNAS.
\end{abstract}

Key words: Anti-mPNAS-4; Polyclonal antibody; Apoptosis-related protein family; One-step affinity purification

*These authors contributed equally to this study.

Research supported by the National Key Basic Research Program of China (\#2004CD518800), National Natural Sciences Foundation of China (\#20505006), and National 863 Projects.

Received January 31, 2008. Accepted May 26, 2008

\section{Introduction}

Apoptosis, programmed cell death, is a genetically regulated, morphologically distinct form of cell death. Apoptotic cells can be recognized by morphology such as smaller nuclei and condensed chromatin, by characteristic molecular features, and by some histochemical techniques such as in situ end labeling. Apoptosis is currently believed to be involved in a wide range of pathologic conditions, including neurodegenerative and cardiovascular diseases, cancer, and autoimmune diseases. However, the mechanism of apoptosis is not clearly understood and therefore it is important to study genes involved in the regulation of this event.

PNAS-4 is a novel apoptosis-related protein family which is highly conserved from vertebrates to invertebrates. All PNAS-4 proteins contain a conserved DUF-862 domain. Recently, Filippov et al. (1) reported that human 
PNAS-4 (hPNAS-4) is a novel pro-apoptotic gene activated during the early response to DNA damage which is thought to play a critical role in cellular function regarding the maintenance of genomic integrity. James et al. (2) reported that the transcript level of hPNAS-4 is greatly upregulated in glucocorticoid-treated human lens epithelial cells (HLE B-3), indicating that hPNAS-4 may be involved in perturbation of lens epithelial cell proliferation and differentiation.

The mouse homolog gene (denoted mPNAS-4), located in the $1 \mathrm{H} 3$ chromosomal region, encodes a 194amino acid residue protein and possesses $96 \%$ identity with the hPNAS-4 protein. Consistent with the high sequence conservation, data obtained in studies by our group (Zhang P, Wang CT, Yan F, Gou LT, Yuan Z, Tong $\mathrm{AP}$, unpublished results) also showed similarity in the biological function between them. We observed that overexpression of mPNAS-4 can promote apoptotic death in lung carcinoma cells (LL2) and colon carcinoma cells (CT26) of Lewis mice both in vitro and in vivo, indicating that mPNAS-4 could be used as a new target for effective therapeutic intervention.

However, many of the properties of PNAS-4, such as its biological activity, are not well understood. Therefore, the expression and the preparation of recombinant mPNAS4 protein (rmPNAS-4) and its polyclonal antibody should provide effective experimental tools for identifying its subcellular localization, expression profile, the interacting proteins, etc. This strategy of studying novel genes at the protein level should facilitate further investigations and is generally accepted worldwide (3-8).

In the present study, the full length mPNAS-4 (21 kDa) fused with glutathione (GST) residues (47 kDa) was expressed in Escherichia coli BL21 (DE3) cells, and this recombinant protein (rmPNAS-4) was purified by one-step affinity purification on a glutathione Sepharose 4B column. The fusion protein was identified by electrospray ionization-mass spectrometry (ESI-MS) analysis and polyclonal antibodies against rmPNAS-4 were prepared. Western blot and immunohistochemistry analysis showed that the polyclonal antibodies were suitable to detect the presence of both the exogenous and endogenous mPNAS-4 effectively.

\section{Material and Methods}

\section{Total RNA isolation}

Total RNA was extracted from $0.1 \mathrm{~g}$ mouse liver tissues using a standard Trizol RNA isolation protocol (Invitrogen, USA). Prior to use, RNA concentrations were quantified by absorbance measurement, and RNA integrity was con- firmed by electrophoresis.

\section{RT-PCR amplification of mPNAS-4 cDNA}

The forward primer was 5'-GCGGATCCATGGCCAA CCAGCCCATCATC-3' and the reverse primer was 5'CCGCTCGAGCTATAGTTTTGTGTGGCGCCCAGG-3'. Sequences of the forward and reverse oligonucleotides contained BamHI and $\mathrm{Xhol}$ restriction sites (underlined), respectively. The primer pairs were designed according to the full-length cDNA sequence of the mPNAS-4 gene in GenBank (GenBank accession \#NM_024282) and used in the reverse transcription-polymerase chain reaction (RTPCR) with a one-step RT-PCR kit (TaKaRa, Japan) according to manufacturer instructions (Figure 1).

\section{Plasmid construction and identification}

The amplified mPNAS-4 cDNA and expression vector pGEX-6P-1 were digested with BamHI and Xhol, respectively, and then ligated overnight at $16^{\circ} \mathrm{C}$, followed by transformation into competent $E$. coli XL1-Blue cells. The positive clones were detected by digestion with $\mathrm{BamHI}$ and Xhol (Figure 3) and confirmed by DNA sequencing.

\section{Expression of the fusion protein}

The expression plasmid pGEX-6P-1-mPNAS-4 was used to transform competent $E$. coli BL21 (DE3) cells. The bacterial cells were cultured in LB broth containing $100 \mu \mathrm{g} /$ $\mathrm{mL}$ ampicillin. When the absorbance at $600 \mathrm{~nm}$ reached 0.6 , isopropyl-ß-D-thiogalactopyranoside (IPTG) was added to the culture at a final concentration of $1 \mathrm{mM}$ for 16 $\mathrm{h}$ at $20^{\circ} \mathrm{C}$ to induce the expression of rmPNAS-4, and then harvested. The cell pellets were resuspended in phosphate-buffered saline (PBS), $\mathrm{pH} 7.4$, and sonicated for 90 $\mathrm{s}$ at $200 \mathrm{~W}$ on ice. The total lysate induced with IPTG was then separated into soluble and insoluble fractions by centrifugation at $800 \mathrm{~g}$ for $25 \mathrm{~min}$ at $4^{\circ} \mathrm{C}$. The expression and solubility of mPNAS-4 were then analyzed in parallel by $12 \%$ SDS-PAGE followed by staining with Coomassie brilliant blue R-250 (Figure 4) at room temperature

\section{Purification of the fusion proteins}

The harvested cell pellets were resuspended in $30 \mathrm{~mL}$ PBS and $1 \mathrm{mM}$ phenylmethylsulfonyl fluoride was added to the slurry and incubated on ice for $30 \mathrm{~min}$. The cells were then sonicated on ice for $40 \mathrm{~min}$ and $20 \%$ deoxycholic acid was added to a final concentration of $2 \%$. After mixing and incubation at room temperature for $10 \mathrm{~min}$, the lysate was centrifuged at $800 \mathrm{~g}$ for $30 \mathrm{~min}$ at $4^{\circ} \mathrm{C}$. The procedures for rmPNAS-4 purification were carried out according to manufacturer instruction. Fractions were collected into $1.0-\mathrm{mL}$ tubes and analyzed by SDS-PAGE (Figure 4). The pres- 
ence of recombinant protein in the eluted fractions was confirmed by Western blot using anti-GST monoclonal antibody with horse radish peroxidase (HRP) conjugates (Sigma, USA) as shown in Figure 5.

\section{mPNAS-4 identification by mass spectrometry}

The purified mPNAS-4 proteins were separated by $12 \%$ SDS-PAGE. The mPNAS- 4 band was excised, and in-gel digestion of proteins was carried out using MS grade Trypsin Gold according to manufacturer instructions. Mass spectra were acquired with an ESI-Q-TOF MS. The MS/ MS data were acquired by the MassLynx software (Micromass, Waters, USA) and converted to PKL files by the ProteinLynx 2.2.5 software (Waters, USA). The pkl files were analyzed using the MASCOT search engine (www.matrixscience.com) (Figure 6).

Preparation of mPNAS-4 polyclonal antibodies and specificity analysis

The purified rmPNAS-4 identified by mass spectrometry was used as an immunogen to raise polyclonal antibodies in New Zealand white rabbit according to reported methods (9). Antibody titer was determined by ELISA. The antiserum was tested by Western blot analysis using the purified rmPNAS-4 and total proteins from mouse brain

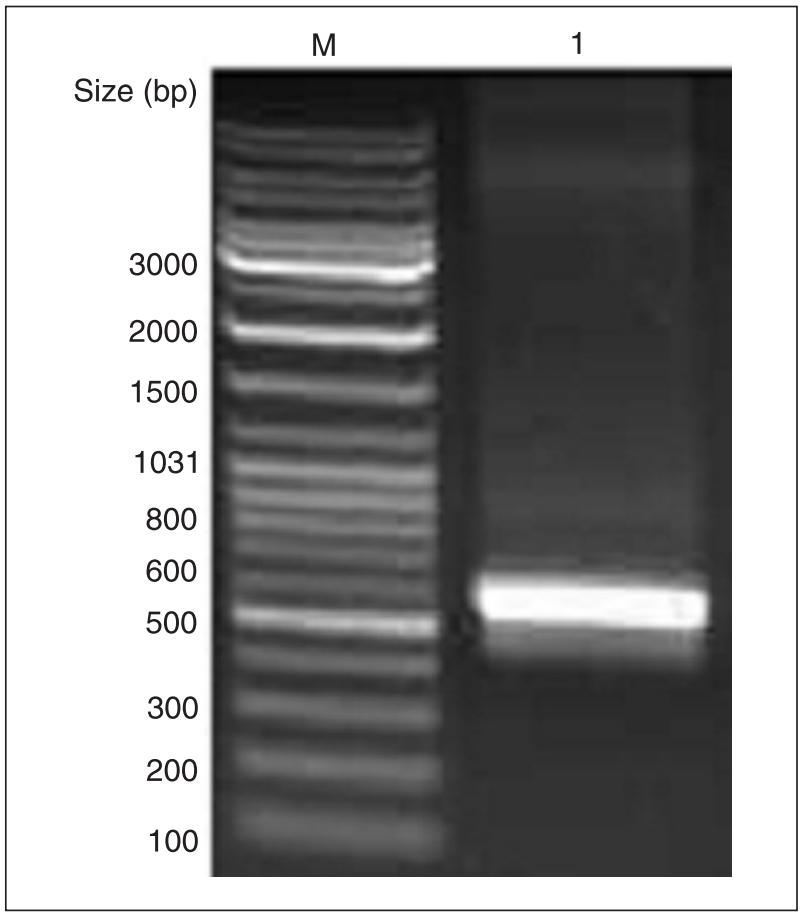

Figure 1. RT-PCR amplification of mPNAS-4 cDNA from the total RNA of mouse liver tissues. $\mathrm{M}=$ DNA marker; lane $1=$ amplified mPNAS-4 cDNA fragment. tissue. Protein concentration was determined by the method of Bradford (10). The protein samples were separated by $12 \%$ SDS-PAGE and electrophoretically transferred onto a polyvinylidene difluoride membrane. After blocking, the polyvinylidene difluoride membranes were incubated with antiserum at 1:4000 dilution for 1-2 $\mathrm{h}$ at room temperature and then incubated in 1:5000 diluted goat anti-rabbit IgG conjugated with HRP for $1 \mathrm{~h}$. After washing three times with Tris-buffered saline with Tween, the membrane was developed using the enchanced chemiluminescence detection kit (Amersham Biosciences, USA). Non-immunized serum was used as the control (Figure 7A).

\section{Cell immunohistochemistry}

To determine whether the polyclonal antibodies were suitable for recognition of the endogenous mPNAS-4 proteins in cells, immunohistochemistry was performed in LL2 cells plated onto coverslips using the labeled streptavidinbiotin method. The anti-mPNAS-4 polyclonal antiserum was used as the primary antibody $(1: 1000)$ and the second antibody was a biotinylated anti-rabbit IgG. The coverslips were then stained with HRP-streptavidin reagents (Dako, USA) and diaminobenzidine and brown staining was considered to be positive (Figure 7C). Non-immunized serum was used as the control (Figure 7B).

Analysis of apoptosis of mPNAS-expressing LL2 cells

Apoptosis in transient mPNAS-4-expressing LL2 cells was analyzed by Hoechst 33258 staining. LL2 cells were seeded onto 6-well plates and, when reaching 70-80\% confluence, were transfected with enhanced green fluorescent protein (EGFP)-N $\mathrm{N}_{1}-\mathrm{mPNAS}-4$ and EGFP-N $\mathrm{N}_{1}$ vector. One day later, GFP fluorescence was detected under an inverted fluorescence microscope to evaluate the transfection efficiency. Cells were then fixed, washed twice with PBS and stained with Hoechst 33258 staining solution according to instructions (Beyotime, China). Stained nuclei were detected under a fluorescence microscope (Figure 8).

\section{Results and Discussion}

\section{Cloning mPNAS-4 cDNA}

To obtain mPNAS-4 gene-specific cDNA fragments, RT-PCR was performed with total RNA from mouse liver tissues. We obtained a fragment of approximately $600 \mathrm{bp}$ in length, as shown in Figure 1, consistent with the expected size.

\section{Homology between mPNAS-4 and hPNAS-4}

The amino acid sequence of mPNAS-4 possessed 
96\% identity with hPNAS-4, suggesting that mPNAS-4 might exert effects similar to those of hPNAS-4 (Figure 2), which has been reported to be a novel pro-apoptotic gene. We found that overexpression of mPNAS-4 resulted in the inhibition of proliferation and the promotion of apoptosis in LL2 cells and CT26 cells in vivo and in vitro (Zhang P, Wang CT, Yan F, Gou LT, Yuan Z, Tong AP, unpublished data).

Identification of the recombinant plasmid pGEX-6P-1mPNAS-4

The purified PCR product was digested with BamHI/ Xhol and cloned into the pGEX-6P-1 vector treated with the corresponding enzymes. The recombinant plasmid pGEX-6P-1-mPNAS-4 was confirmed correctly by PCR, by digestion with BamHI/Xhol (Figure 3) and DNA sequencing.

Expression of biologically active recombinant eukaryotic proteins in bacteria is difficult to achieve due to improper folding and poor solubility $(11,12)$. The GST Gene Fusion System is one of the four main prokaryotic expression systems available. As the good fusion partner protein,
GST could produce correctly folded and soluble heterologous proteins in bacterial cytoplasm $(13,14)$. Thus, we selected pGEX-6P-1 containing the GST tag at the $\mathrm{N}$ terminus to express mPNAS-4.

\section{mPNAS-4 expression}

IPTG were added to the E. coli BL21 (DE3) cells at a final concentration of $1 \mathrm{mM}$ for $16 \mathrm{~h}$ at $20^{\circ} \mathrm{C}$ to induce the expression of rmPNAS-4. SDS-PAGE analysis revealed that the fusion protein with the GST tag at the N-terminal was about $47 \mathrm{kDa}$ (Figure 4, lanes 2 and 3), which was consistent with the predicted molecular weight (containing GST tag sequences) and was present almost exclusively in soluble form (Figure 4, lane 3).

Temperature was one of the key factors for the induction of eukaryotic proteins in bacteria. Because of rapid expression at high temperature, error folding usually occurred and the induced protein was in the form of an inclusion body. In our studies, we first induced the recombinant protein at $37^{\circ} \mathrm{C}$ and most of it was insoluble. To obtain the soluble protein, we later changed the induction temperature. Ultimately, we obtained satisfactory results
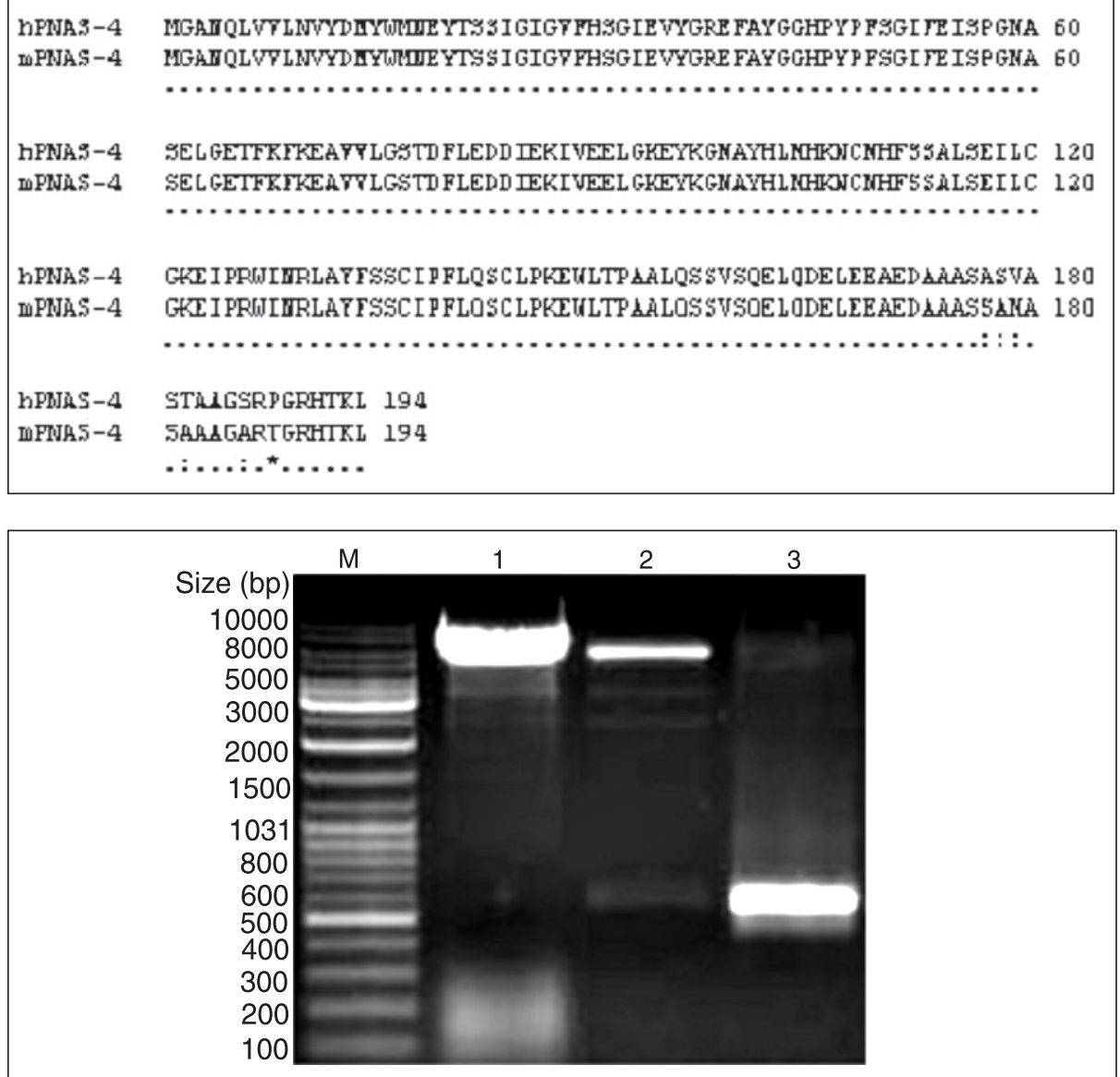

Figure 2. Sequence alignment of hPNAS-4 and mPNAS-4. Identical residues in the two sequences are indicated by a dot, conserved residues by a colon, and similar residues by a star. The alignment was done using the CLUSTAL W software, version 1.83 .

Figure 3. Identification of $p G E X-$ 6P-1-mPNAS-4 by PCR and BamHI/Xhol digestion. $\mathrm{M}=\mathrm{DNA}$ marker; lane 1, plamid pGEX6P-1; lane 2, plasmid pGEX-6P1-mPNAS-4 digested with BamHI/Xhol; lane 3, PCR product with plasmid pGEX-6P-1mPNAS-4 as template. 
at $20^{\circ} \mathrm{C}$ which were useful for further study.

\section{Purification of mPNAS-4}

The rmPNAS-4 protein was purified by one-step affinity purification using glutathione Sepharose $4 B$ as described in Refs. 15-17. The presence of recombinant protein in the eluted fractions was confirmed by SDS-PAGE and Western blot using an anti-GST monoclonal antibody with HRP conjugates (Figure 5).

The PNAS-4 gene was first reported (1) at the 96th Annual Meeting of AACR in 2005 by Filippov, who reported that PNAS-4 might be involved in the apoptotic response to DNA damage. James et al. (2) later reported that PNAS-4 may be acting by disturbing lens epithelial cell proliferation and differentiation. Furthermore, Filippov et al. (1) reported that hPNAS-4 was a novel pro-apoptotic gene able to promote apoptosis in osteosarcoma U2OS cells in vivo. The mPNAS-4 protein possessed a high identity with the hPNAS-4 protein, and therefore we believed that mPNAS4 could play an important role in cell proliferation and differentiation as we had found in our studies. However, the molecular mechanisms by which mPNAS-4 acted remain unknown. Therefore, with a better understanding of mPNAS-4, the purified soluble fusion protein and the specific antibody could open up new possibilities in many applications such as molecular immunology, the production of vaccines and studies involving protein-protein and DNA-protein interactions.

mPNAS-4 identification by ESI-MS analysis

The three representative peptide fragments in Figure 6 were unambiguously matched to the trypsin-digested ions of mPNAS-4, indicating that our expressed and purified protein rmPNAS-4 were completely correct.

One of the peptide fragments obtained by ESI-MS was the DUF-862 domain with high conservatism in the PNAS4 protein family and having $100 \%$ identity with hPNAS-4. These data indicated that the DUF-862 domain would be involved in the biological function of the PNAS-4.

Production of polyclonal antibodies against the recombinant protein and analysis of their specificity

The specificity of rabbit antiserum was determined by Western blot analysis using purified rmPNAS-4 and mPNAS-4 from mouse brain tissue as antigens. There were two positive bands at positions of 21 and $47 \mathrm{kDa}$, respectively (Figure 7A). However, non-immunized serum was negative. Immunohistochemistry using the antimPNAS-4 polyclonal antiserum in LL2 cells revealed strong brown staining in the cytoplasm, excluding the nuclei (Figure $7 \mathrm{C}$ ). However, the controls showed no staining in the cytoplasm (Figure 7B). These results indicate that our rabbit antiserum specifically and effectively recognized both exogenous recombinant mPNAS-4 and endogenous mPNAS-4 in tissues and cells.

Apoptosis induced by overexpression of mPNAS-4

To determine if mPNAS-4 would promote apoptosis,

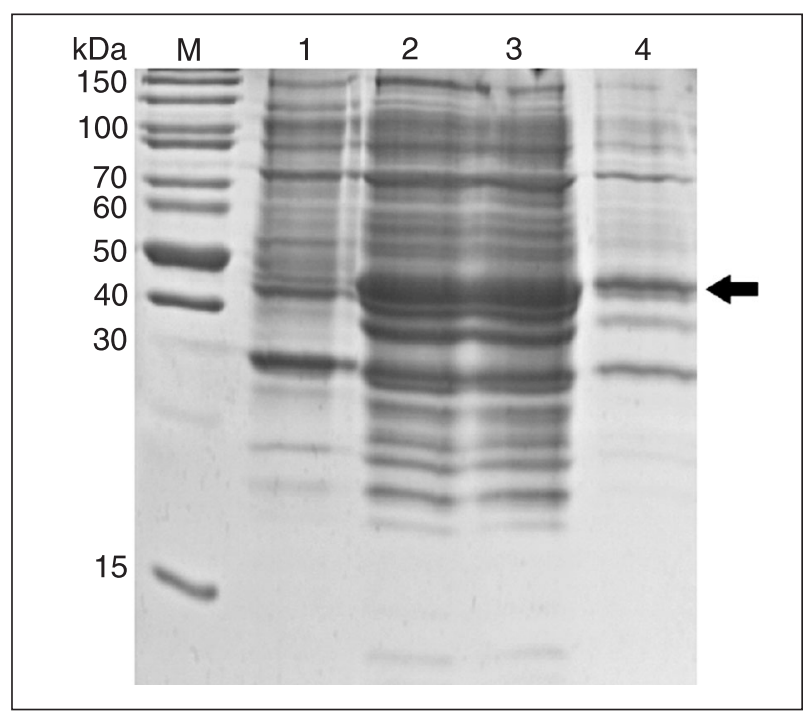

Figure 4. Expression and identification of the recombinant protein. $\mathrm{M}=$ protein molecular weight markers; lane 1, Escherichia coli BL21 (DE3)/pGEX-6P-1-mPNAS-4 whole cell proteins before induction; lane 2, E. coli BL21 (DE3)/pGEX-6P-1-mPNAS-4 whole cell proteins after induction; lane 3 , soluble fraction from induced cell lysates; lane 4, insoluble fraction from induced cell lysates.

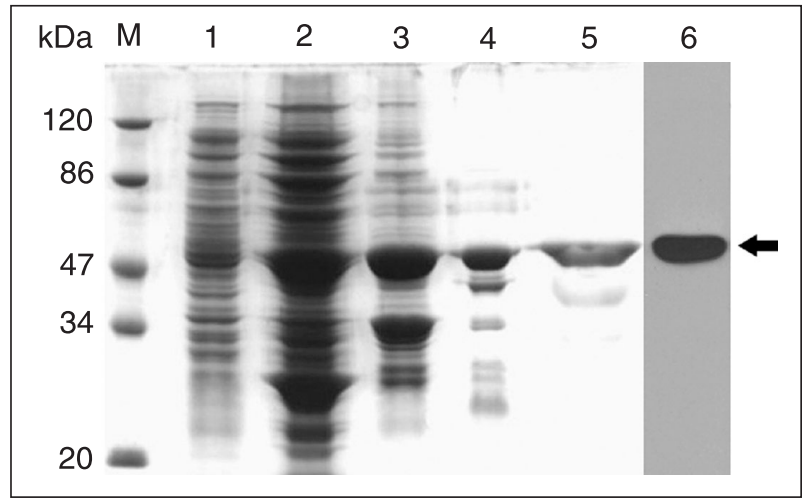

Figure 5. Purification of mPNAS-4. $M$ = protein molecular weight markers. SDS-PAGE (12\%) analysis of the crude extracts of Escherichia coli BL21 before and after induction with $1 \mathrm{mM}$ isopropyl- $ß-D$-thiogalactopyranoside at $20^{\circ} \mathrm{C}$ (lanes 1,2 ) and affinity chromatography-purified mPNAS-4 from the first to the third tube of elution (lanes 3-5). Western blot analysis with an anti-glutathione-tag $\mathrm{mAb}$ (lane 6). 

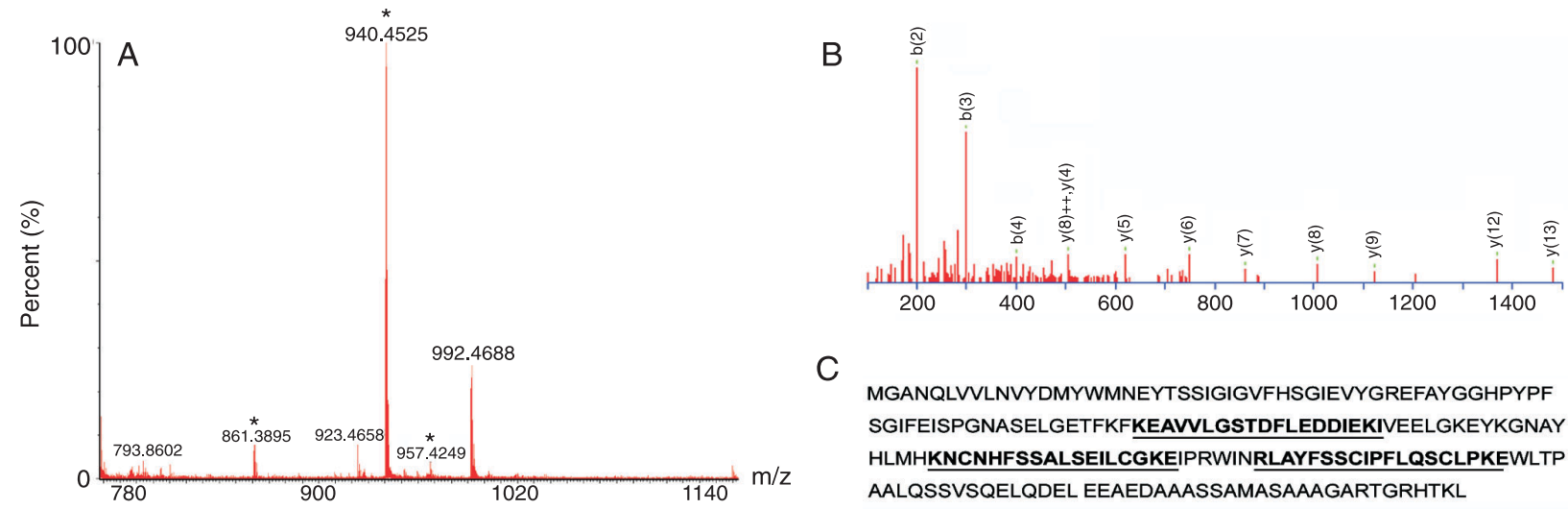
C
MGANQLVVLNVYDMYWMNEYTSSIGIGVFHSGIEVYGREFAYGGHPYPF SGIFEISPGNASELGETFKFKEAVVLGSTDFLEDDIEKIVEELGKEYKGNAY HLMHKNCNHFSSALSEILCGKEIPRWINRLAYFSSCIPFLQSCLPKEWLTP AALQSSVSQELQDEL EEAEDAAASSAMASAAAGARTGRHTKL

Figure 6. Electrospray ionization-mass spectrometry (ESI-MS) identification of tryptic peptides from the purified recombinant mPNAS-4 protein. A, ESI-MS of mPNAS-4 showing 3 peptides (asterisks) that could be sequence. The search parameters of the parent and fragment mass error tolerance were set at 0.1 and $0.05 \mathrm{kDa}$, respectively. $B$, An example of an MS/MS spectrum of the parent ion 940.4525 . $C$, Output of the database search by the MASCOT program using MS/MS data resulted in the identification of mPNAS-4. The three peptide sequences of mPNAS-4 are shown in bold and underlined.
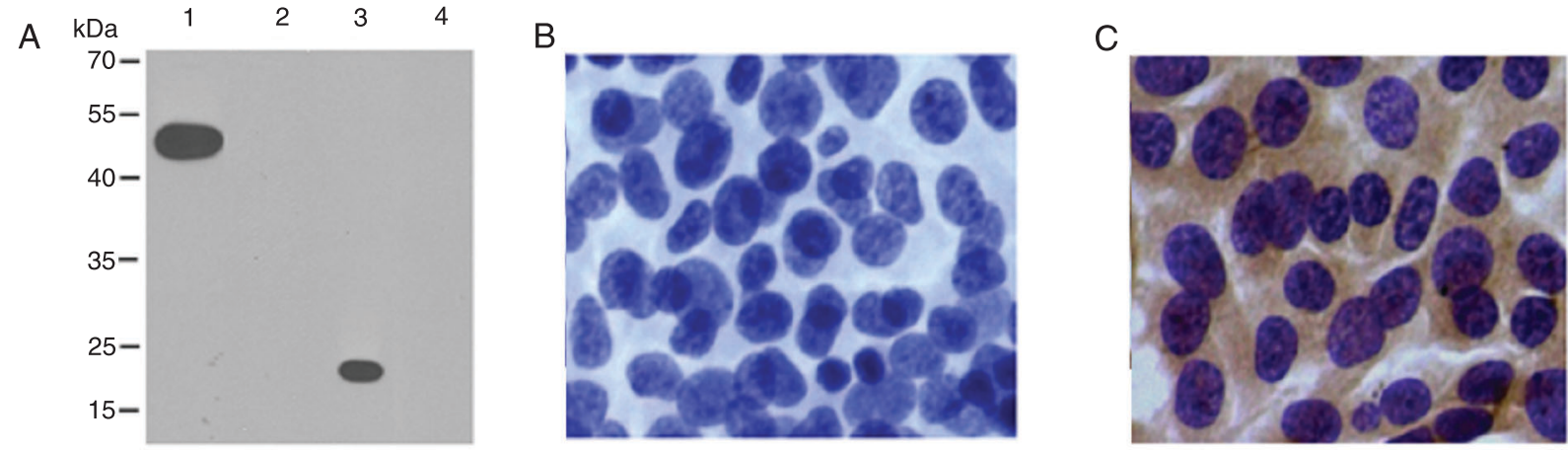

Figure 7. Western blot and immunostaining analysis of mPNAS-4 expression using rabbit antiserum. $A$, Immunized serum (1:4000) was used to detect the total protein from rmPNAS-4 (lane 1) and mPNAS-4 (lane 3). Non-immunized serum was used as control (lanes 2 and 4). Samples (100-300 ng) were loaded onto the gel. Horse radish peroxidase-conjugated goat anti-rabbit lgG (1:5000) and enchanced chemiluminescence were used for color development. $B$, The immunohistochemistry assay of LL2 cells used the antiserum (1:1000). Cell nuclei were identified by staining and observed under a microscope (200X). The polyclonal anti-mPNAS-4 antibodies can recognize the native mPNAS-4 protein in the cytoplasm (C), in agreement with the subcellular location of enhanced green fluorescent protein-mPNAS-4. Brown staining (C) showed positive results, while hematoxylin staining showed the negative results of the controls $(\mathrm{B})$.
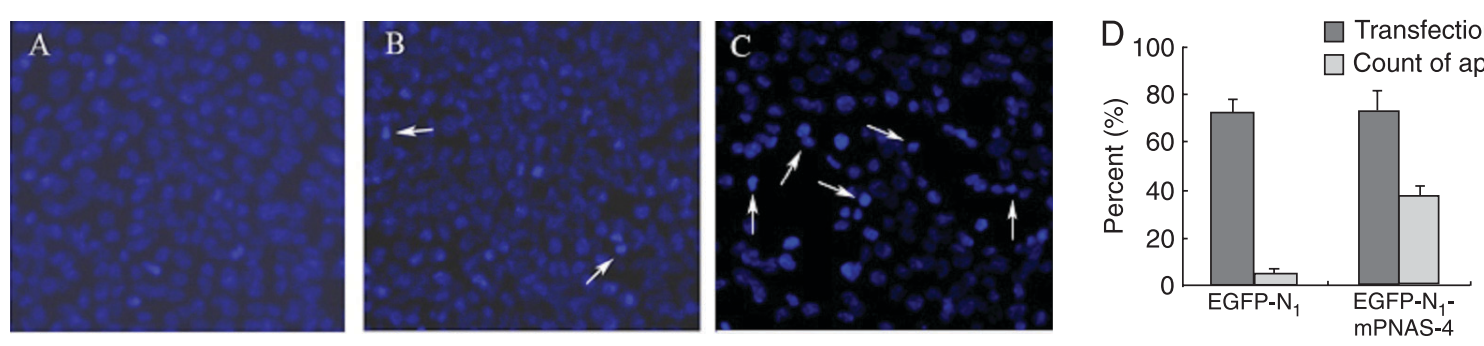

Figure 8. Hoechst 33258 staining of LL2 cells transfected with mPNAS-4. After $24 \mathrm{~h}$, LL2 cells treated with enhanced green fluorescent protein (EGFP)- $\mathrm{N}_{1}-\mathrm{mPNAS}-4$ and EGFP- $\mathrm{N}_{1}$ vector were stained with Hoechst 33258 . Nuclei were visualized by fluorescence microscopy (200X). A, Wild-type LL2 cells. B, LL2 cells transfected with EGFP-N 1 vector. C, LL2 cells overexpressed with mPNAS-4. The arrows indicate the presence of apoptotic nuclear profiles. The quantitative analysis of these results is shown in $D$. The transfection efficiency of the two groups transfected with plasmid was about $70 \%$, but apoptotic cells were much more numerous in the experimental group. 
LL2 cells were stained with Hoechst 33258, 24 h after transient transfection. More nuclei of LL2 cells transfected with EGFP-N $\mathrm{N}_{1}$-mPNAS-4 appeared to be hypercondensed (brightly stained; Figure $8 \mathrm{C}$ ) compared to control cells (Figure 8A,B). Furthermore, GFP fluorescence showed that about $70 \%$ of LL2 cells were transfected into both groups treated with the plasmid. The quantitative analysis of the transfection efficiency and apoptosis of cells from the two groups is shown in Figure 8D. Recombinant eukaryotic proteins overexpressed in cells are likely to be biologically active in their natural conformation. In our previous studies (Yuan Z, Yan F, Zhang P, Wang CT, Gou LT, Zhao XY, Wei YQ, unpublished results), mPNAS-4 was also transfected into murine fibroblast NIH3T3 cells, but no obvious change was detected even after $72 \mathrm{~h}$ compared to control. Therefore, the present results indicate that the larger number of apoptotic cells found in the experimental group can be attributed mostly to overexpression of mPNAS-4.

Because of its $96 \%$ identity with hPNAS-4, mPNAS-4 was also thought to be involved in the process of apopto- sis. This hypothesis was supported by our studies, although the mechanism is still unknown.

As a member of a novel apoptosis-related protein family, mPNAS-4 is thought to play an important role during tumorigenesis and tumor development. Therefore, it was essential to identify how mPNAS-4 performs its function. The purified rmPNAS-4 with GST-tag would undoubtedly be useful to investigate the proteins interacting with it by co-immunoprecipitation, tandem affinity purification-based MS technology and GST pull-down analysis (18-21). And the antibody produced will also serve as a tool to explore the function of mPNAS-4, permitting the identification of its potential binding partners, the study of its structure and its mechanism of pathogenesis, and the progression of cancer cells.

\section{Acknowledgments}

The authors thank Dr. Xin-yu Zhao and Prof. Bei-Meng Sun for helpful discussions and correction of the English language.

\section{References}

1. Filippov V, Filippova M, Sinha D, DuerksenHughes PJ. PNAS-4: A Novel Pro-Apoptotic Gene Activated During the Early Response to DNA Damage. Proceedings of the American Association for Cancer Research. 96th Annual Meeting. Anaheim, 2005.

2. James ER, Fresco VM, Robertson LL. Glucocorticoid-induced changes in the global gene expression of lens epithelial cells. J Ocul Pharmacol Ther 2005; 21: 11-27.

3. Guo F, Li SQ, Chu YH, Huang XF, Sun LM, Li YQ, et al. High-level expression, polyclonal antibody preparation and sub-cellular localization analysis of mouse Rhox 5 protein. Protein Expr Purif 2007; 54: 247-252.

4. Rautureau G, Jouvensal L, Decoville M, Locker D, Vovelle $\mathrm{F}$, Schoentgen F. Cloning, high yield over-expression, purification, and characterization of CG18594, a new PEBP/ RKIP family member from Drosophila melanogaster. Protein Expr Purif 2006; 48: 90-97.

5. Park HJ, Jeon JH, Kang SG, Lee JH, Lee SA, Kim HK. Functional expression and refolding of new alkaline esterase, EM2L8 from deep-sea sediment metagenome. Protein Expr Purif 2007; 52: 340-347.

6. Londer YY, Pokkuluri PR, Erickson J, Orshonsky V, Schiffer $M$. Heterologous expression of hexaheme fragments of a multidomain cytochrome from Geobacter sulfurreducens representing a novel class of cytochromes c. Protein Expr Purif 2005; 39: 254-260.

7. Menard LP, Lussier JG, Lepine F, Paiva de SC, Dubreuil JD. Expression, purification, and biochemical characterization of enteroaggregative Escherichia coli heat-stable enterotoxin 1. Protein Expr Purif 2004; 33: 223-231.

8. Li A, Kato Z, Ohnishi H, Hashimoto K, Matsukuma E, Omoya $\mathrm{K}$, et al. Optimized gene synthesis and high expression of human interleukin-18. Protein Expr Purif 2003; 32: 110-118.

9. Voss EW Jr, Miklasz SD, Petrossian A, Dombrink-Kurtzman MA. Polyclonal antibodies specific for liganded active site (metatype) of a high affinity anti-hapten monoclonal antibody. Mol Immunol 1988; 25: 751-759.

10. Bradford MM. A rapid and sensitive method for the quantitation of microgram quantities of protein utilizing the principle of protein-dye binding. Anal Biochem 1976; 72: 248-254.

11. LaVallie ER, McCoy JM. Gene fusion expression systems in Escherichia coli. Curr Opin Biotechnol 1995; 6: 501-506.

12. Smith DB, Johnson KS. Single-step purification of polypeptides expressed in Escherichia coli as fusions with glutathione S-transferase. Gene 1988; 67: 31-40.

13. Stevens RC. Design of high-throughput methods of protein production for structural biology. Structure 2000; 8: R177R185.

14. Guzzo CM, Yang DC. Systematic analysis of fusion and affinity tags using human aspartyl-tRNA synthetase expressed in E. coli. Protein Expr Purif 2007; 54: 166-175.

15. Kovacs-Nolan J, Sasaki E, Yoo D, Mine Y. Cloning and expression of human rotavirus spike protein, VP8*, in Escherichia coli. Biochem Biophys Res Commun 2001; 282: 1183-1188.

16. Smith DB. Generating fusions to glutathione S-transferase for protein studies. Methods Enzymol 2000; 326: 254-270.

17. Lundberg $M$, Holmgren $A$, Johansson $M$. Human glutaredoxin 2 affinity tag for recombinant peptide and protein purification. Protein Expr Purif 2006; 45: 37-42. 
18. Yan J, Yang XP, Kim YS, Joo JH, Jetten AM. RAP80 interacts with the SUMO-conjugating enzyme UBC9 and is a novel target for sumoylation. Biochem Biophys Res Commun 2007; 362: 132-138.

19. Huang X, Shi Z, Wang W, Bai J, Chen Z, Xu J, et al. Identification and characterization of a novel protein ISOC2 that interacts with p16INK4a. Biochem Biophys Res Commun 2007; 361: 287-293.
20. Quaedackers ME, van den Brink CE, van der Saag PT, Tertoolen LG. Direct interaction between estrogen receptor alpha and NF-kappaB in the nucleus of living cells. Mol Cell Endocrinol 2007; 273: 42-50.

21. Nian H, Fan C, Liao S, Shi Y, Zhang K, Liu Y, et al. RNF151, a testis-specific RING finger protein, interacts with dysbindin. Arch Biochem Biophys 2007; 465: 157-163. 